\title{
DESMAME dA VENTILAÇÃO MECÂNICA dE PACIENTES CRÍTICOS ADULTOS: REVISÃO SOBRE A UTILIZAÇÃO DE PROTOCOLOS
}

\author{
MECHANICAL VENTILATION WEANING ON CRITICALLY ILL ADULT PATIENTS: \\ A REVIEW OF PROTOCOLS USE
}

\author{
Robson Inácio Marinho \\ Robson Alves da Silva \\ Catharinne Angélica Carvalho de Farias \\ Joelson dos Santos Silva \\ Lívia Carla Bezerra de Macêdo \\ Caroline Ferreira Schön \\ Vitória Jéssica Teixeira Dantas \\ Fagna Maria de Andrade e Silva \\ Robson Inácio Marinho \\ E-mail: robson_inacio15@hotmail.com \\ Hospital Universitário Onofre Lopes \\ Natal/RN
}

\section{RESUMO}

Objetivo: Analisar os efeitos de protocolos de desmame da Ventilação Mecânica (VM) em pacientes críticos. Método: Tratou-se de uma revisão de literatura realizada nas bases de dados Lilacs, SciELO e MEDLINE, envolvendo artigos publicados entre 2012 e 2017, utilizando-se os descritores "protocolo", "ventilação mecânica" e "desmame" e suas variantes na língua inglesa, além do descritor boleriano "AND". Resultados: Foram encontrados 156 artigos respeitando-se os critérios de inclusão, restando 10 artigos após aplicação dos critérios de exclusão. A maioria dos artigos encontrados (80\%) estavam escritos em língua inglesa, $60 \%$ dos artigos realizaram TRE com tubo T e a maioria dos trabalhos utilizou como preditores de sucesso, principalmente, o Índice de Respiração Rápida e Superficial e a pressão inspiratória máxima. Conclusões: A utilização de protocolos de desmame para liberação da VM de pacientes críticos adultos reduz taxas de reintubação e da falha de desmame e diminui a duração da VM, com consequente redução nas complicações decorrentes da VM prolongada.

Palavras-chave: Unidades de terapia intensiva; Extubação; Ventilação mecânica; Desmame do respirador. 


\section{ABSTRACT}

Objective: To analyze the effects of weaning protocols of MV on critical patients. Methods: It was a literature review carried out in the Lilacs, SciELO and MEDLINE databases, involving articles published between 2012 and 2017, using the descriptors "protocol", "mechanical ventilation", and "weaning" and their variants in the Portuguese language, besides the Boolean descriptor "AND". Results: We found 156 articles respecting the inclusion criteria, remaining 10 articles after applying the exclusion criteria. Most of the articles found (80\%) were written in English, $60 \%$ of articles performed T-tube SBT, and most of the articles used as predictors of success, mainly, the Rapid and Shallow Breathing Index and the maximum inspiratory pressure. Conclusion: The use of weaning protocols to release MV from critically ill adult patients reduces reintubation rates, weaning failure rates, and decreases MV duration, with consequent reduction in complications resulting from prolonged MV.

Keywords: Intensive care units; Airway extubation; Respiration, Artificial; Ventilator weaning.

\section{INTRODUÇÃO}

A ventilação mecânica invasiva (VM) é considerada uma ferramenta com propósito de salvar a vida de pacientes instáveis e criticamente doentes ${ }^{1,2}$, apresentando-se necessária à maioria dos pacientes internados em unidades de terapia intensiva (UTI) ${ }^{2}$. Apesar de seu objetivo principal ser trazer conforto e melhora aos pacientes com insuficiência respiratória aguda (IRpA) ou crônica agudizada, a VM também traz vários riscos aos pacientes ${ }^{3,4}$, como o desenvolvimento de pneumonia associada à ventilação (PAV), lesão pulmonar induzida pela ventilação e disfunção diafragmática induzida pela ventilação, além de comprovadamente aumentar a taxa de mortalidade dos pacientes ${ }^{4,5}$, se prolongada por tempo superior às necessidades deles.

O processo de transição da respiração por suporte ventilatório mecânico até a capacidade de respirar espontaneamente é definido como desmame da ventilação mecânica. Estudos mostram que o desmame ocupa cerca de $40 \%$ do tempo total da $\mathrm{VM}^{2,6}$ e que deve ser realizado com maior exatidão e o mais precocemente possível ante a estabilização clínica do paciente, a qual resumidamente envolve a resolução do quadro que levou o indivíduo à intubação endotraqueal, nível de consciência adequado, estabilidade hemodinâmica e capacidade de respirar em modos espontâneos de VM, sem apresentar sinais de desconforto.

Depois de alcançados esses objetivos, a aptidão à liberação da VM é avaliada por um teste de respiração espontânea (TRE), o qual pode ser realizado utilizando-se um Tubo $\mathrm{T}$ associado à oxigenação suplementar (sem suporte pressórico) ou ainda conectado ao ventilador mecânico, nos modos CPAP ou PSV, com parâmetros pressóricos considerados baixos ${ }^{4}$.

Nos dias atuais, o desmame da VM é dividido em três tipos, de acordo com o número de TRE necessários para a extubação: desmame simples (o paciente necessita de apenas 1 
TRE); desmame difícil (o paciente necessita de até 3 TRE ou de até 7 dias após o primeiro teste); e desmame prolongado (o paciente necessita de mais de 3 TRE ou mais de 7 dias após a realização do primeiro teste $)^{4,6,7}$. Diversas variáveis (também conhecidas como índices ou preditores) podem ser utilizadas para guiar e auxiliar na avaliação de pacientes submetidos ao desmame da VM. Entre elas, as mais abordadas pela literatura são: o volume corrente (VT), volume minuto (VE), pressão inspiratória máxima $\left(\mathrm{PI}_{\text {máx }}\right)$, frequência respiratória (f), índice de respiração rápida e superficial (IRRS) e o índice integrativo de desmame (IWI)2.

Nos últimos anos, muitos trabalhos vêm mostrando que os desmames de VM direcionados por protocolos trazem uma variedade de benefícios aos pacientes críticos, tais como redução no tempo de $\mathrm{VM}^{8-11}$, redução no índice de falha de desmame ${ }^{10,12}$, redução no número de falhas de extubação ${ }^{13}$ (necessidade de reintubação em menos de 48 horas após extubação) e redução no tempo de internação em UTI e hospitalar ${ }^{3}$. No entanto, alguns profissionais de saúde ainda parecem resistentes à utilização de protocolos para o desmame da VM e optam por se manter sob a subjetividade da avaliação clínica dos pacientes de forma isolada.

Diante do exposto, este trabalho tem como objetivo principal elaborar uma revisão bibliográfica sobre a utilização de protocolos para desmame da ventilação mecânica, abordando os principais índices de sucesso de desmame, bem como desfechos acerca da mortalidade, tempo de VM e taxa de sucesso do desmame.

\section{MÉTODO}

O presente estudo se tratou de uma revisão de literatura de trabalhos que utilizaram protocolos de desmame da VM em pacientes críticos adultos. Para isso, foram realizadas buscas de artigos publicados entre janeiro de 2012 e novembro de 2017 nas bases de dados SciELO, LILACS e MEDLINE/ PubMed, utilizando os descritores "desmame", "ventilação mecânica" e "protocolo", além de seus equivalentes na língua inglesa: "weaning", "mechanical ventilation" e "protocol", em conjunto com o termo de busca Booleano "AND".

Os critérios de inclusão para escolha dos artigos presentes nesta revisão foram: artigos publicados nos últimos cinco anos que abordassem a aplicação de protocolos para desmame da ventilação mecânica, nos idiomas português, espanhol e inglês. Foram excluídos do estudo artigos que tratassem do desmame da VM em crianças e neonatos, trabalhos envolvendo pacientes traqueostomizados, trabalhos incompletos ou com restrição de acesso, além de duplicatas e artigos de revisão e meta-análises.

A pesquisa bibliográfica foi feita por um único autor, que realizou todas as etapas, desde o processo de busca dos artigos científicos até a leitura dos trabalhos selecionados. 


\section{RESULTADOS}

Após pesquisa dos artigos utilizando-se os termos de busca já citados, foi encontrado um total de 156 trabalhos publicados no período determinado. De acordo com os critérios de exclusão, 5 artigos foram excluídos por serem duplicatas, e mais 73 foram excluídos após leitura dos títulos dos trabalhos, restando 78 resumos para serem lidos. Após a leitura dos resumos, outros 38 artigos foram excluídos da revisão, além de mais 4 artigos pela impossibilidade de acesso.

Diante disso, 36 artigos foram selecionados para leitura na íntegra, resultando na seleção final de 10 artigos para serem incluídos, analisados e discutidos nesta revisão (figura 1), dos quais 5 avaliaram pacientes sob VM com duração $\geq 48$ horas, 3 com pacientes sob VMI $\geq 24$ horas, enquanto 2 não especificaram o tempo de VM. Em relação ao tipo de paciente, 3 trabalhos foram realizados com pacientes cirúrgicos, 4 com pacientes clínicos e cirúrgicos, 1 com pacientes com diagnóstico de doença pulmonar obstrutiva crônica (DPOC) e 2 não especificaram.

Dos artigos incluídos neste trabalho, $80 \%$ foram escritos em língua inglesa, enquanto os outros $20 \%$ se dividiram equitativamente em língua portuguesa e espanhola. A maioria dos trabalhos $(60 \%)$ executou o TRE dos pacientes submetidos à VM por meio do Tubo T associado à oxigenoterapia, enquanto os demais foram realizados por meio do modo PSV, apresentando discretas diferenças nos níveis de pressão de suporte (PS) utilizados (PS entre 5 e $7 \mathrm{cmH} 2 \mathrm{O}$; PEEP entre 5 e $10 \mathrm{cmH}$ 2O) (tabela 1). A frequência absoluta de utilização de índices preditores de desmame da VM está exposta na figura 2, na qual se pode notar uma preferência pelo uso do Índice de Respiração Rápida e Superficial (IRRS) e pela Pressão inspiratória máxima ( $\mathrm{PI}_{\text {máx }}$ ).

\section{DISCUSSÃO}

O presente estudo reuniu e analisou artigos científicos sobre a importância da utilização de protocolos para guiar e fortalecer decisões acerca da retirada de pacientes críticos do suporte ventilatório invasivo, bem como mostrou os índices preditores de desmame mais utilizados na literatura, de acordo com os descritores de busca utilizados neste trabalho.

Em sua maioria, os artigos analisados mostraram redução nas taxas de reintubação ${ }^{13}$, redução no tempo de $\mathrm{VM}^{8-11}$ e maiores taxas de sucesso de desmame ${ }^{10,12}$. No entanto, alguns achados na literatura apresentam resultados mais discretos, enquanto outros chegam até a mostrar resultados desfavoráveis em grupos específicos de pacientes, como visto em um trabalho que avaliou o impacto de um protocolo de desmame em pacientes neurocirúrgicos ${ }^{18}$, o qual não obteve diferença estatística no sucesso do desmame nem na duração da VM. Por outro lado, quando se associou o nível de consciência de pacientes neurológicos sob VM à utilização de um protocolo de desmame, obteve-se redução no tempo de VM e aumento na taxa de sucesso de desmame ${ }^{19}$. 
Figura 1: Fluxograma da seleção dos artigos

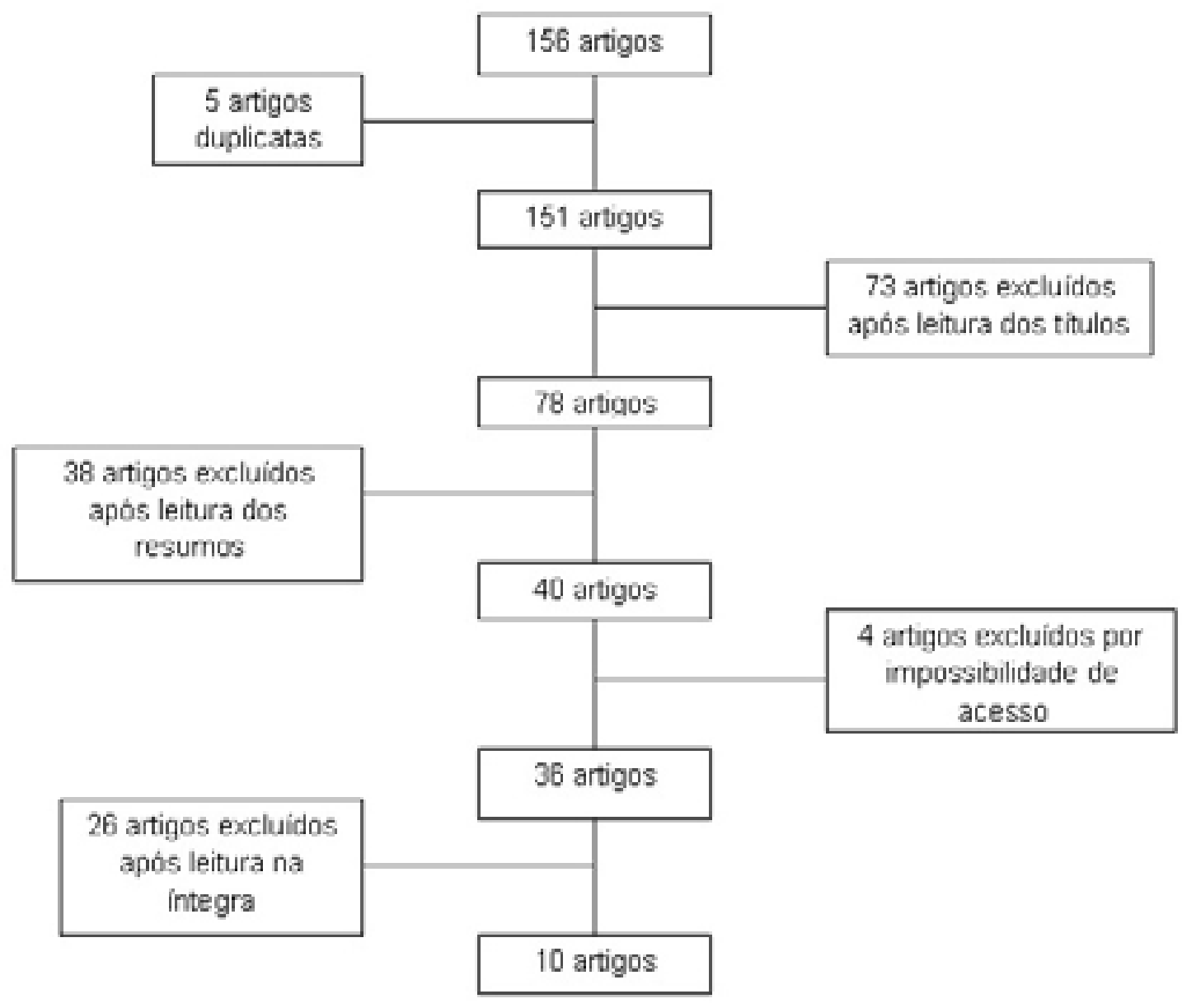

Figura 2: Frequência da utilização de preditores de desmame

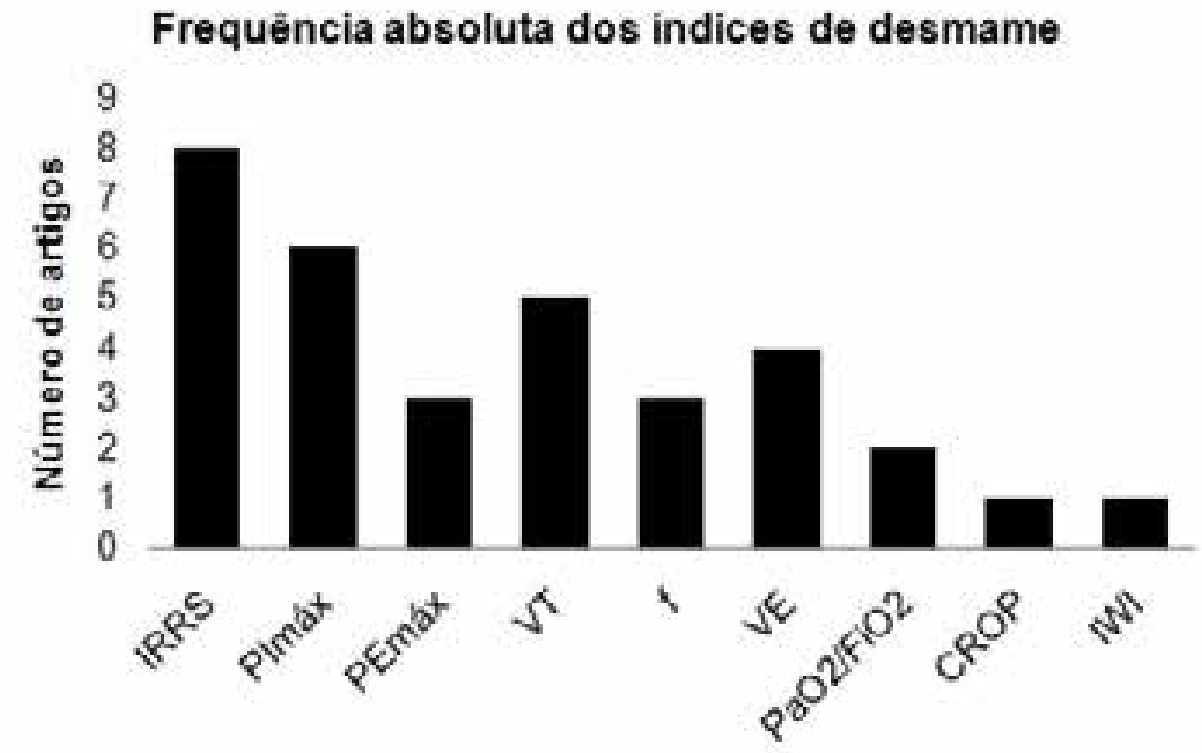

Preditores de desmame

IRRS: Índice de Respiração Rápida e Superficial; $\mathrm{PI}_{\text {maxx }}$ : Pressão Inspiratória máxima; $\mathrm{PE}_{\text {máx }}$ : Pressão Expiratória máxima; VT: Volume corrente; f: Frequência respiratória; VE: Volume minuto; $\mathrm{PaO}_{2} / \mathrm{FiO}_{2}$ : Índice de oxigenação; $\mathrm{CROP}$ : Complacência, frequência, oxigenação, pressão; IWI: İndice integrativo de desmame. 
Tabela 1: Artigos incluídos na revisão após aplicação dos critérios

\begin{tabular}{|c|c|c|c|c|c|}
\hline Autor(es)/Ano & Objetivo & Descrição & $\begin{array}{c}\text { Particularidades } \\
\text { do TRE e da } \\
\text { extubação }\end{array}$ & $\begin{array}{l}\text { Principais } \\
\text { índices de } \\
\text { sucesso de } \\
\text { desmame }\end{array}$ & Resultados \\
\hline $\begin{array}{l}\text { Teixeira C, Maccari } \\
\text { JG, Vieira'SRR, } \\
\text { Oliveira RP, Savi A, } \\
\text { Machado AS et al. } \\
2012\end{array}$ & $\begin{array}{l}\text { Avaliar o uso suplementar de } \\
\text { um protocolo cujo objetivo é } \\
\text { ajudar na acurácia do julgamento } \\
\text { clinico isolado na decisão sobre o } \\
\text { desmame da VM. }\end{array}$ & $\begin{array}{l}\text { Estudo clínico prospectivo } \\
\text { multicentrico realizado entre } \\
\text { janeiro de } 2002 \text { e dezembro de } \\
2005 \text { com pacientes clinicos e } \\
\text { cirúrgicos de desmame difícil, sob } \\
\text { VM por mais de } 48 \text { horas. } n=731\end{array}$ & \begin{tabular}{|l|} 
Tubo T \\
VNI preventiva nos \\
grupos de risco.
\end{tabular} & $\begin{array}{l}I R R S<105 \\
\mathrm{PI}_{\text {máx }}<-30\end{array}$ & $\begin{array}{l}\text { Redução na taxa } \\
\text { de reintubação } \\
\text { de pacientes de } \\
\text { dificil desmame. }\end{array}$ \\
\hline $\begin{array}{l}\text { Silva CSM, } \\
\text { Timenetsky KT, } \\
\text { Taniguchi C, } \\
\text { Calegaro S,Azevedo } \\
\text { CSA, Stus R et al. } \\
2012\end{array}$ & $\begin{array}{l}\text { Descrever a aplicação de } \\
\text { um protocolo de desmame } \\
\text { e os resultados do seu uso } \\
\text { relacionados ao tempo de VM e } \\
\text { necessidade de reintubação. }\end{array}$ & $\begin{array}{l}\text { Estudo retrospectivo dos dados } \\
\text { obtidos de pacientes clinicos } \\
\text { e cirúrgicos entre janeiro de } \\
2009 \text { e abril de } 2010 \text {, os quais } \\
\text { necessitavam de VM por mais de } \\
24 \text { horas. } n=252\end{array}$ & $\begin{array}{l}\text { PSV (PS: 5-7; PEEP: } \\
\text { 5-10; FiO2: 0,3-0,4) } \\
\text { Duração: } 30-120 \text { min. } \\
\text { Cuff-leak test. } \\
\text { VNI preventiva nos } \\
\text { grupos de risco. }\end{array}$ & $\begin{array}{l}P I_{\text {máx }} \\
P E_{\text {máx }} \\
V T \\
V E \\
I R R S<104\end{array}$ & $\begin{array}{l}\text { Redução no } \\
\text { tempo de VMi } \\
\text { taxas aceitáaieis } \\
\text { de reintubação. }\end{array}$ \\
\hline $\begin{array}{l}\text { Savi A, Teixeira C } \\
\text { Silva JM, Borges LG, } \\
\text { Pereira PA, Pinto KB } \\
\text { et al. 2012 }\end{array}$ & $\begin{array}{l}\text { Avaliar o potencial de preditores } \\
\text { de desmame para o sucesso } \\
\text { na extubação de pacientes com } \\
\text { desmame simples, sob VMM. }\end{array}$ & $\begin{array}{l}\text { Ensaio clínico multicêntrico, } \\
\text { prospectivo realizado em } 3 \\
\text { UTIs cirúrgicas, com pacientes } \\
\text { sob VM por mais de } 48 \text { horas, } \\
\text { internados entre janeiro de } 2003 \\
\text { e dezembro de } 2007 . n=500\end{array}$ & \begin{tabular}{|l|} 
Tubo T com $\mathrm{O}_{2}$ \\
suplementar para manter \\
SpO $\geq 90 \%$ (máximo de \\
$8 \mathrm{~L} / \mathrm{min}$ ). \\
Duração: 30 minutos.
\end{tabular} & $\begin{array}{l}\text { IRRS } \\
\text { CROP } \\
\mathrm{PI}_{\text {máx }} \\
\mathrm{PE}_{\text {máx }} \\
\mathrm{PaO}_{2} / \mathrm{FiO}_{2} \\
\mathrm{f} \\
\mathrm{VT}\end{array}$ & $\begin{array}{l}\text { Nenhum preditor } \\
\text { foi identificado } \\
\text { como indice } \\
\text { de falha do } \\
\text { desmame } \\
\text { simples. }\end{array}$ \\
\hline $\begin{array}{l}\text { González IMM, } \\
\text { Chelala BEF, } \\
\text { Portelles AC, Reyes } \\
\text { KP, Trujillo AG. } 2014\end{array}$ & $\begin{array}{l}\text { Avaliar o impacto da } \\
\text { implementação de um protocolo } \\
\text { de desmana sobre o tempo de } \\
\text { duração da vi e a mortalidade } \\
\text { entre os pacientes ventilados. }\end{array}$ & $\begin{array}{l}\text { Estudo quase experimental com } \\
\text { pacientes graves sob vM por } \\
\text { mais de } 24 \text { horas. } n=135\end{array}$ & $\begin{array}{l}\text { Tubo T. } \\
\text { Duração: } 60 \mathrm{~min} .\end{array}$ & $\begin{array}{l}\text { IRRS }<105(<65 \\
\text { anos }) \\
\text { IRRS }<95(\geq 65 \\
\text { anos })\end{array}$ & $\begin{array}{l}\text { Discreta redução } \\
\text { na mortalidadade } \\
\text { e redução no } \\
\text { tempo de VM. }\end{array}$ \\
\hline $\begin{array}{l}\text { Kirakli C, Ediboglu O, } \\
\text { Naz I, Cimen P, Tatar } \\
\text { D. } 2014\end{array}$ & $\begin{array}{l}\text { Avaliar o impacto de um } \\
\text { protocolo sobre o tempo de } \\
\text { VM, tempo de desmame, } \\
\text { permanencia na UTI, taxa } \\
\text { de sucesso de desmame e } \\
\text { mortalidade de pacientes com } \\
\text { DPOC. }\end{array}$ & $\begin{array}{l}\text { Estudo tipo coorte que incluiu } \\
\text { pacientes com DPOC que } \\
\text { necessitaram de VM por mais de } \\
48 \text { horas devido exacerbação. } \\
\mathrm{n}=170 \text {. }\end{array}$ & $\begin{array}{l}\text { Tubo T. } \\
120 \text { min. } \\
\text { PSV (PS: 7) após } 3 \\
\text { falhas no TRE com } \\
\text { Tubo T. }\end{array}$ & Não especificado. & $\begin{array}{l}\text { Melhora no } \\
\text { sucesso do } \\
\text { desmame e } \\
\text { redução no } \\
\text { tempo de VM. }\end{array}$ \\
\hline $\begin{array}{l}\text { Gupta P, Giehler } \\
\text { K, Walters RW, } \\
\text { Meyerink K, } \\
\text { Modrykamien AM. } \\
\text { 2014 }\end{array}$ & $\begin{array}{l}\text { Comparar o efeito da aplicação } \\
\text { de um protocolo de desmame da } \\
\text { VM em pacientes com desmame } \\
\text { simples e dificili. }\end{array}$ & $\begin{array}{l}\text { Ensaio clínico retrospectivo } \\
\text { realizado na UTl mista de } \\
\text { um hospital universitário de } \\
\text { Creighton, Nebraska, EUA. n } \\
=803 .\end{array}$ & $\begin{array}{l}\text { PSV (PS: 5; PEEP: 5). } \\
\text { Duração: } 60 \text { min. }\end{array}$ & $\begin{array}{l}\mathrm{VT}>5 \mathrm{~mL} / \mathrm{Kg} \\
\mathrm{PI}_{\text {max }}<-20 \\
\mathrm{IRRS}^{\mathrm{R}}<105 \\
\mathrm{VE}<15 \mathrm{~L} / \mathrm{min}\end{array}$ & $\begin{array}{l}\text { Aumentou o } \\
\text { número de dias } \\
\text { fora da vM } \\
\text { nos pacientes } \\
\text { com desmame } \\
\text { simples e difícil. }\end{array}$ \\
\hline $\begin{array}{l}\text { Jones K, Newhouse } \\
\text { R, Johnson K, Seidl } \\
\text { K. } 2014\end{array}$ & $\begin{array}{l}\text { Avaliar resultados subsequentes } \\
\text { à implementação de protocolos } \\
\text { de despertar espontâneo e teste } \\
\text { de respiração espontânea em } \\
\text { pacientes criticos adultos sob } \\
\text { VM. }\end{array}$ & $\begin{array}{l}\text { Estudo retrospectivo secundário } \\
\text { com pacientes criticos (clínicos } \\
\text { e cirurgicos) avaliados entre } \\
\text { novembro de } 2009 \text { e dezembro } \\
\text { de } 2010 . n=112 \text {. }\end{array}$ & PSV & Não especificado. & $\begin{array}{l}\text { Reduziu o tempo } \\
\text { de VM sem } \\
\text { aumentar a taxa } \\
\text { de extubação } \\
\text { não programada. }\end{array}$ \\
\hline $\begin{array}{l}\text { El-Baraday GF, El- } \\
\text { Shmaa NS, Ganna } \\
\text { SA. } 2015\end{array}$ & $\begin{array}{l}\text { Avaliar a eficiência do IWI } \\
\text { como preditor de sucesso de } \\
\text { desmame. }\end{array}$ & $\begin{array}{l}\text { Estudo controlado, randomizado, } \\
\text { prospectivo, com observador } \\
\text { cego. Avaliou pacientes sob VM } \\
\text { por mais de } 24 \text { horas, de janeiro } \\
\text { de } 2014 \text { a abril de } 2015 . n=120 \text {. }\end{array}$ & $\begin{array}{l}\text { PSV (PS: 5). } \\
\text { Duração: } 120 \text { min. }\end{array}$ & $\begin{array}{l}\mathrm{PaO}_{2} / \mathrm{FiO}_{2} \geq 200 \\
\mathrm{f}<30 \mathrm{irpm} \\
\mathrm{VT}>5 \mathrm{~mL} / \mathrm{Kg} \\
\mathrm{VE}<15 \mathrm{~L} / \mathrm{min} \\
\mathrm{IRRS}<105 \\
\mathrm{IWI}>25\end{array}$ & $\begin{array}{l}\text { Confirmou a } \\
\text { utilidade do } \\
\text { IWl durante } \\
\text { o processo } \\
\text { de desmame, } \\
\text { sendo efetivo } \\
\text { em predizer } \\
\text { sucesso e falha } \\
\text { de desmame. }\end{array}$ \\
\hline
\end{tabular}


Tabela 1: Artigos incluídos na revisão após aplicação dos critérios

\begin{tabular}{|c|c|c|c|c|c|}
\hline Autor(es)/Ano & Objetivo & Descrição & $\begin{array}{l}\text { Particularidades } \\
\text { do TRE e da } \\
\text { extubação }\end{array}$ & $\begin{array}{l}\text { Principais } \\
\text { índices de } \\
\text { sucesso de } \\
\text { desmame }\end{array}$ & Resultados \\
\hline $\begin{array}{l}\text { Bien US, Souza } \\
\text { GF, Campos ES, } \\
\text { Carvalho EF, } \\
\text { Fernandes MG, } \\
\text { Santoro I et al. } 2015\end{array}$ & $\begin{array}{l}\text { Avaliar a acurácia da } \mathrm{PI}_{\text {máx }} \mathrm{e} \\
\text { do IRRS como preditores para } \\
\text { o sucesso do desmame de } \\
\text { pacientes sob VM. }\end{array}$ & $\begin{array}{l}\text { Estudo transversal prospectivo } \\
\text { com pacientes clinicos e } \\
\text { cirúrgicos sob VMicor por mais de } 24 \\
\text { h, executado entre julho de } 2011 \\
\text { e julho de } 2013 \text { em uma UTI } \\
\text { adulto, localizada em São Paulo, } \\
\text { Brasil. no }=195 \text {. }\end{array}$ & $\begin{array}{l}\text { Tubo T }(5 \text { a } 10 \mathrm{~L} / \mathrm{min} \text { de } \\
\text { O }{ }^{2} \text { para manter } \mathrm{SpO}_{2} \\
>90 \% . \\
\text { Duração: } 30 \mathrm{~min} .\end{array}$ & $\begin{array}{l}\text { VE } \\
\mathrm{f} \\
\text { VTe } \\
\text { IRRS }<100 \\
\mathrm{PI}_{\text {máx }}\end{array}$ & $\begin{array}{l}\text { PI e IRRS } \\
\text { dirétamente } \\
\text { ligados ao } \\
\text { sucesso do } \\
\text { desmame da VM. }\end{array}$ \\
\hline $\begin{array}{l}\text { Borges LGA, Savi A, } \\
\text { Teixeira C, Oliveira } \\
\text { RP, Camillis MLF, } \\
\text { Wickert R et al. } 2017\end{array}$ & $\begin{array}{l}\text { Avaliar a aceitação e o uso } \\
\text { institucional de um protocolo de } \\
\text { desmame da vM e avaliar a taxa } \\
\text { de sucesso do desmame. }\end{array}$ & $\begin{array}{l}\text { Ensaio clínico de coorte } \\
\text { prospectiva realizado com } \\
\text { pacientes sob VM por mais de } \\
24 \text { horas, internados na UTI } \\
\text { cirúrgica do Hospital Moinho de } \\
\text { Ventos, conduzido por mais de } \\
7 \text { anose dividido em } 3 \text { fases: } \\
\text { desenvoluvimento, aplicação e } \\
\text { monitorização. } n=2469 \text {. }\end{array}$ & $\begin{array}{l}\text { Tubo T. } \\
\text { Duração: 30-120 min. }\end{array}$ & $\begin{array}{l}\text { IRRS }<105 \\
\mathrm{PI}_{\text {max }}<-30 \\
\mathrm{PE}_{\text {max }}\end{array}$ & $\begin{array}{l}\text { Melhorou a taxa } \\
\text { de sucesso de } \\
\text { desmame da VM. }\end{array}$ \\
\hline
\end{tabular}

Tabela apresentando as principais informações acerca dos artigos analisados neste trabalho. TRE: Teste de respiração espontânea; VM: Ventilação mecânica invasiva; VNI: Ventilação não-invasiva; IRRS: Índice de respiração rápida e superficial; $\mathrm{PE}_{\mathrm{MÁx}}$ : Pressão expiratória máxima; VT: Volume corrente; VE: Volume minuto; UTI:Unidade de terapia intensiva; DPOC: Doença pulmonar obstrutiva crônica; $\mathrm{SpO}_{2}$ : Saturação periférica de oxigênio; VTe: Volume corrente expirado; CROP: Índice de Complacência respiratória, frequência respiratória, oxigenação e pressão; $\mathrm{PaO}_{2} / \mathrm{FiO}_{2}$ : Índice de oxigenação; IWI: Índice integrativo de desmame.

No guideline de prática clínica acerca da retirada do suporte ventilatório mecânico invasivo em pacientes críticos adultos publicado em 2017, uma das perguntas respondidas foi sobre a utilização de protocolos de desmame de $\mathrm{VM}^{20}$. Os resultados mais importantes desse guia foram redução de 25 horas no tempo de duração da VM e redução de um dia no tempo de internação na UTI, sem grandes impactos na taxa de mortalidade. Devido aos resultados tímidos e às limitações metodológicas da maioria dos estudos encontrados na literatura, a recomendação do uso de protocolos para desmame é condicional, e o grau de evidência é baixo ${ }^{20}$. No entanto, a padronização de uma ferramenta para auxiliar em uma decisão clínica tão importante como o desmame da VM deve ser encorajada e continuamente estudada para benefício da população e dos profissionais de saúde.

Atrelada aos protocolos de desmame da VM, encontra-se, na maioria dos estudos, a aplicação de preditores de sucesso de desmame e extubação. Nos artigos relacionados nesta revisão, os preditores mais frequentes foram o IRRS e a $\mathrm{PI}_{\text {máx }}$ O IRRS, ou Índice de Tobin ${ }^{21}$, é entendido como a razão entre f e VT em litros e faz uma estimativa da capacidade do paciente em manter VT e VE dentro de uma f adequada, avaliando indiretamente complacência pulmonar e resistência da musculatura inspiratória. Nesta revisão, o IRRS foi o preditor mais prevalente , $^{8,9,12-17}$ entre os 
estudos, e o ponto de corte mais utilizado para sucesso de desmame foi IRRS $<105$.

Apesar de o IRRS estimar resistência muscular adequada para manter a respiração espontânea por períodos indeterminados, ele não é capaz de estimar a força muscular inspiratória. Para isso, muitos trabalhos realizam avaliação da $\mathrm{PI}_{\text {máx }}$ como preditor de sucesso de desmame da $\mathrm{VM}^{8,12-15,17}$. O ponto de corte mais prevalente foi de $\mathrm{PI}_{\text {máx }}<-30$, ou seja, valores menos negativos indicam maior capacidade de negativação de pressão e melhor força muscular inspiratória.

Dos cinco trabalhos que especificaram a forma de aferição da $\mathrm{PI}_{\text {máx }}$, quatro a fizeram utilizando manovacuômetro analógico associado a uma válvula unidirecional. Contudo, existem relatos na literatura de estudos que utilizaram manovacuômetros digitais para obtenção desse índice em pacientes sob desmame da $\mathrm{VM}^{17,23}$.

Uma das causas mais comuns das falhas de extubação é o desenvolvimento de edema laríngeo ${ }^{25}$, clinicamente comprovado por meio do estridor laríngeo após extubação. $O$ desenvolvimento do estridor é mais prevalente em pacientes do sexo feminino, pacientes com tubo orotraqueal (TOT) de maior diâmetro, idade superior a 70 anos e tempo de VM superior a 96 horas $^{20,24,25}$. O cuff-leak test, ou teste de vazamento do balonete, vem-se tornando um importante preditor do desenvolvimento de edema laríngeo após extubação, com sensibilidade diretamente proporcional ao tempo de $\mathrm{VM}^{24,25}$. Apesar disso, apenas um artigo presente nesta revisão incluiu esse teste em seu protocolo de desmame e extubação ${ }^{8}$, o que vai de encontro às recomendações mais atuais encontradas na literatura, as quais sugerem a realização do cuff-leak test nos pacientes que se encontram nos grupos de risco para o desenvolvimento de edema laríngeo, bem como indica a administração de esteroides sistêmicos ao menos 4 horas antes da extubação em pacientes que falharam no teste ${ }^{20}$.

Outro ponto importante a ser avaliado é a utilização da ventilação não-invasiva (VNI) em sua ação preventiva após extubação, a qual esteve presente no protocolo de apenas quatro dos dez artigos analisados neste trabalho $0^{8,12-14}$, tendo sido utilizada em pacientes com DPOC ${ }^{8,12-}$ ${ }^{14}$, insuficiência cardíaca congestiva (ICC $)^{8,12-}$ ${ }^{14}$, síndrome do desconforto respiratório agudo $(\mathrm{SDRA})^{13}$ e estridor laríngeo ${ }^{12}$.

Em comparação à utilização de máscara de Venturi após extubação, o uso profilático da VNI pode diminuir o tempo de permanência na UTI, bem como a taxa de insuficiência respiratória após extubação em pacientes submetidos à VM por mais de 48 horas $^{26}$. Guias atuais de prática clínica recomendam fortemente o uso de VNI profilática, principalmente em pacientes com hipercapnia, DPOC e ICC, com o objetivo de reduzir as complicações decorrentes da VM e da internação na UTI, desde que seu uso seja aplicado imediatamente após extubação ${ }^{27}$. 


\section{CONCLUSÕES}

A utilização de protocolos de desmame para liberação da VM de pacientes críticos adultos, em associação com a aplicação de preditores de sucesso do desmame, vem mostrando resultados importantes no campo da terapia intensiva, como redução nas taxas de reintubação, de desmame e na duração da VM, com consequente diminuição nas complicações decorrentes da VM prolongada.

Dentre os preditores de sucesso da VM, destacam-se, na literatura, o IRRS e a $\mathrm{PI}_{\text {máx }}$, presentes na maioria dos estudos aqui abordados.

\section{REFERÊNCIAS}

1. Juern JS. Removing the critically ill patient from mechanical ventilation. Surg Clin N Am. 2012; 92:1475-1483.

2. Pérez-Calatayud AA, Carrillo-Esper R, Arch-Tirado E. Propuesta de evaluación cuantitativa em el protocolo ultrasonográfico para retiro de la ventilación mecânica invasiva (GMEMI score). Gac Med Mex. 2016;1 52:304-312.

3. Ward D, Fulbrook P. Nursing strategies for effective weaning of the critically ill mechanically ventilated patient. Crit Care Nurs Clin N Am. 2016; 28:499-512.

4. Tallo FS, Sandre VL, Lopes RD, Simone SVCA, Lopes AC. Weaning from mechanical ventilation: approach for the internist. Rev Soc Bras Clin Med. 2014; 12(1):57-63.

5. Chen L, Gilstrap D, Cox CE. Mechanical ventilator discontinuation process. Clin Chest Med. 2016; 37:693-699.

6. Jeong BH, Ko MG, Nam J, Yoo H, Chung CR, Suh GY, et al. Differences in clinical outcomes according to weaning classifications in medical intensive care units. PLoS ONE. 2015; 10(4):e0122810. doi:10.1371/journal.pone.0122810.

7. Associação de Medicina Intensiva Brasileira, Sociedade Brasileira de Pneumologia e Tisiologia, 2013. Diretrizes Brasileiras de Ventilação Mecânica, Brasil; 2013.

8. Silva CSM, Timenetsky KT, Taniguchi C, Calegaro S, Azevedo CSA, Stus R, et al. Low mechanical ventilation times and reintubation rates associated with a specific weaning protocol in an intensive care unit setting: a retrospective study. CLINICS. 2012;67(9):995-1000.

9. González IMM, Chelala BEF, Portelles AC, Reyes KP, Trujillo AG. Implementación de un protocolo para la separación de la ventilación mecânica de pacientes graves, dirigido por enfermeros intensivistas. CCM. 2014; 18(3):430-443.

10. Kirakli C, Ediboglu O, Naz I, Cimen P, Tatar D. Effectiveness and safety of a protocolized mechanical ventilation and weaning strategy of COPD patients by respiratory therapists. J Thorac Dis. 2014; 6(9):1180-1186. 
11. Jones K, Newhouse R, Johnson K, Seidl K. Achieving quality health outcomes through the implementation of a spontaneous awakening and spontaneous breathing trial protocol. AACN Advanced Critical Care. 2014;2 5(1):33-42.

12. Borges LGA, Savi A, Teixeira C, Oliveira RP, Camillis MLF, Wickert R et al. Mechanical ventilation weaning protocol improves medical adherence and results. Journal of Critical Care. 2017; 41:296-302.

13. Teixeira C, Maccari JG, Vieira SRR, Oliveira RP, Savi A, Machado AS, et al. Impacto de um protocolo de ventilação mecânica na taxa de falha de extubação em pacientes de difícil desmame. J Bras Pneumol. 2012; 38(3):364-371.

14. Savi A, Teixeira C, Silva JM, Borges LG, Pereira PA, Pinto KB, et al. Weaning predictors do not predict extubation failure in simple-to-wean patients. Journal of Critical Care. 2012; 27:221-228.

15. Gupta P, Giehler K, Walters RW, Meyerink K, Modrykamien AM. The effect of a mechanical ventilation discontinuation protocol in patients with simple and difficult weaning: impact on clinical outcomes. Respiratory Care. 2014; 59(2):170-177.

16. El-Baraday GF, El-Shmaa NS, Ganna SA. Can integrative weaning index be a routine predictor for weaning success? Indian J Crit Care Med. 2015;1 9(12):703-707.

17. Bien US, Souza GF, Campos ES, Carvalho EF, Fernandes MG, Santoro I, et al. Maximum inspiratory pressure and rapid shallow breathing index as predictors of successful ventilator weaning. J Phys Ther Sci. 2015; 27:3723-3727.

18. Namen AM, Ely EW, Tatter SB, Case LD, Lucia MA, Smith A, et al. Predictors of successful extubation in neurosurgical patients. Am J Resp Crit Care Med. 2001; 163:658-664.

19. Fan L, Su Y, Elmadhoun OA, Zhang Y, Zhang Y, Gao D, et al. Protocol-directed weaning from mechanical ventilation in neurological patients: a randomized controlled trial and subgroup analyses based on consciousness. Neurological Research. 2015; 37(11):1006-1014.

20. Girard TD, Alhazzany W, Kress JP, Ouellette DR, Schmidt GA, Truwit JD. An official American Thoracic Society/American College of Chest Physicians clinical practice guideline: liberation from mechanical ventilation in critically ill adults - Rehabilitation protocols, Ventilator liberation protocols and Cuff leak tests. Am J Respir Crit Care Med. 2017; 195(1):120-133. 
21. Tobin MJ, Jenouri G, Birch S, Lind B, Gonzalez H, Ahmed T, et al. Effect of positive end-expiratory pressure on breathing patterns of normal subjects and intubated patients with respiratory failure. Crit Care Med. 1983; 11(11):859-867.

22. Yang KL, Tobin MJ. A prospective study of indexes predicting the outcome of trials of weaning from mechanical ventilation. 1991; 324(21):1445-1450.

23. Souza LC, Silva Jr CT, Almeida JR, Lugon JR. Comparison of maximum inspiratory pressure, tracheal airway occlusion pressure, and its ratio in the prediction of weaning outcome: impact of the use of a digital vacuometer and the unidirectional valve. Respiratory Care. 2012; 57(8):12851290.

24. Saback LMP, Vieira GF, Costa MD. O uso do teste de escape do balonete como fator preditor de laringoespasmo. Revista Brasileira de Terapia Intensiva. 2008; 20(1):77-81.

25. Wittekamp BHJ, van Mook WNKA, Tjan DHT, Zwveling JH, Bergmans DCJJ. Clinical review: Post-extubation laryngeal edema and extubation failure in critically ill adult patients. Critical Care. 2009; 13(6):233-241.

26. Adryeke E, Ozgultekin A, Turan G, Iskender A, Canpolat G, Pektas A et al. Ventilação mecânica não invasiva após desmame bem-sucedido: uma comparação com a máscara de Venturi. Rev Bras Anestesiol. 2016; 66(6):572-576.

27. Ouellette DR, Patel S, Girard TD, Morris PE, Schmidt GA, Truwit JD, et al. An official American Thoracic Society/American College of Chest Physicians clinical practice guideline: liberation from mechanical ventilation in critically ill adults - Inspiratory pressure aumengtation during spontaneous breathing trials, protocols minimizing sedation and Noinvasive ventilation immediately after extubation. CHEST. 2017; 151(1):166-180.

28. Sessler CN, Grap MJ, Brophy GM. Multidisciplinary management of sedation and analgesia in critical care. Semin Respir Crit Care Med. 2001; 22(2):211-226. 